IAU Colloquium 190 on Magnetic Cataclysmic Variables

ASP Conference Series, Vol. 315, 2004

Sonja Vrielmann 83 Mark Cropper, eds.

\title{
The XMM-Newton-MSSL survey of Polars
}

\author{
Gavin Ramsay, Mark Cropper \\ Mullard Space Science Laboratory, University College London, \\ Holmbury St. Mary, Dorking, Surrey, RH5 6NT, UK
}

\begin{abstract}
The XMM-Newton-MSSL survey of polars is a snapshot survey of nearly 40 polars. We present the initial results, with an emphasis on the energy balance in these systems and the number of systems which were observed in a low accretion state. In contrast to the ROSAT results we find that the vast majority of polars show no 'soft X-ray excess'. We find that half of our sample were observed in low or off accretion states. We find no evidence that these systems were biased towards certain orbital periods.
\end{abstract}

\section{Introduction}

XMM-Newton was launched in 1999 December. It has the largest effective area of any X-ray imaging satellite. It was also the first X-ray mission to have an on-board optical/UV telescope. XMM-Newton has 3 identical X-ray telescopes: one feeds an imaging CCD (EPIC pn) detector operating from $\sim 0.15-10 \mathrm{keV}$. The other two telescopes feed both an imaging CCD detector (EPIC MOS, $\sim 0.15-10 \mathrm{keV}$ ) and a reflection grating spectrometer (RGS, $\sim 0.4-2.4 \mathrm{keV}$ ). The optical/UV telescope (OM) has a diameter of $30 \mathrm{~cm}$ and has a series of filters extending from the $V$ band down to $\sim 2000 \AA$. It has several modes of operation including an imaging and a fast mode which enables a time resolution less than a second. See Jansen et al. (2001) for an overview of the mission and first science results.

\section{The survey}

We have carried out a survey of polars using XMM-Newton. Currently, 33 polars have been observed as part of this survey, with another 6 more systems still to be observed. Three systems were observed as part of the performance verification phase of the mission. One system, RX J2115-58 was the subject of a series of pointings (c.f. Cropper, Ramsay \& Marsh, this volume). Figure 1 shows the orbital period distribution of all known polars together with those systems currently observed as part of our survey. The period distribution of those polars in our survey is comparable with the observed distribution.

The observation length of each polar was typically $\sim 5-6 \mathrm{ksec}$. This was long enough so that we obtained full orbital coverage for many systems. We obtained fast mode observations in the $V$, UVW1 (2400-3400 $\AA$ ) and UVW2 (1800-2400 $)$ 


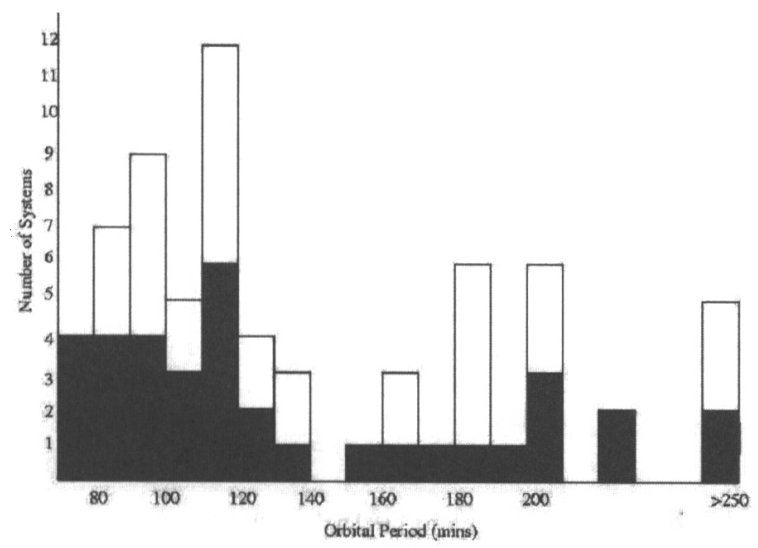

Figure 1. The orbital period distribution of polars together with those currently observed as part of the XMM-Newton-MSSL polar survey.

filters using the optical/UV telescope. Most systems were too faint to obtain useful spectra using the RGS. There are a great many aspects that such a survey can address. Here, we discuss the light curves of individual polars, then provide an initial view of the issue of the 'soft X-ray excess' and examine the implications of the fraction of polars which were in a low accretion state.

\section{Light curves}

Systems which have only one visible accreting pole are particularly interesting in that they allow us to probe the structure of the accretion region as it rotates into view. One example is WW Hor where we directly measure a change in the X-ray hardness ratio which is due to the top of the accretion column first coming into view with the lower layers later (Pandel et al. 2002). Simultaneous $B$ band data using the OM show a quasi-sinusoidal modulation originating from the photosphere of the white dwarf and near-simultaneous $R$ band data obtained from SAAO show flux originating from the cyclotron emission. These data are particularly good at showing the multi-wave band and multi-emission processes seen in polars.

Other examples of one-pole systems include EV UMa and GG Leo which both show prominent accretion stream dips in soft X-rays (and in the UV in the case of GG Leo). These have allowed us to place constraints on the radius and total column density in the stream (Ramsay \& Cropper 2003). All these systems previously mentioned show prominent flux above $1 \mathrm{keV}$. In complete contrast EU UMa shows a low count rate above $1 \mathrm{keV}$, and very little evidence for the presence of a shock.

One unusual aspect of the observations of BY Cam was that the hard Xrays trailed the soft X-rays and are anti-correlated (Ramsay \& Cropper 2002). This may imply that dense blobs of material impact the white dwarf and release 


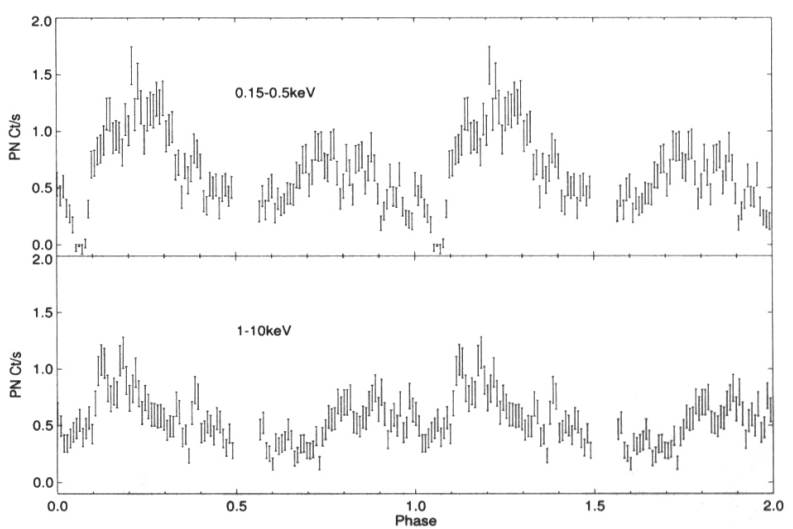

Figure 2. The X-ray data of AN UMa taken using the EPIC pn of the polar AN UMa. The top panel shows the soft X-ray light while the lower shows the hard X-ray light curve.

a sufficient amount of optically thick material to momentarily obscure the hard X-rays.

Timing studies of the eclipse of DP Leo (Pandel et al. 2002) show that the spin period is slightly shorter than the orbital period and the orbital period is decreasing much faster than expected for energy loss by gravitational radiation alone. This is consistent with a separate study of DP Leo by Schwope et al. (2002).

In the next section we discuss the energy balance of AN UMa using XMMNewton and ROSAT data. An example of the general quality of the XMMNewton X-ray data, we show the soft and hard X-ray light curves of AN UMa using the EPIC pn detector in Figure 2.

\section{The energy balance}

For polars in a high accretion state the accretion flow forms a strong shock at some height above the photosphere of the white dwarf. The maximum temperature in the shock is set by the mass of the white dwarf. For a $0.7 M_{\odot}$ white dwarf the shock temperature is $\sim 30 \mathrm{keV}$, with the temperature decreasing as the gas settles onto the white dwarf. Some fraction of the hard X-rays intercept the photosphere of the white dwarf, are thermalised and then re-radiated as soft $\mathrm{X}$-rays or in the extreme UV. The standard model of the shock region predicts $L_{\text {re-processed }} / L_{\text {shock }} \sim 0.5$ (eg Lamb \& Masters 1979).

Observations made using EXOSAT showed that a number of polars showed a large 'soft X-ray excess': assuming the re-processed component was emitted as soft X-rays, the ratio, $L_{r e-p r o c e s s e d} / L_{\text {shock}}$, was well in excess of that predicted by the standard model. Observations made using ROSAT largely supported this view. Ramsay et al. (1994) showed that when the cyclotron emission was included (which contributes to $L_{\text {shock}}$ ) and when bolometric unabsorbed luminosities were used, a significant number of systems showed large soft X-ray excesses. Using unabsorbed fluxes in the ROSAT band (0.1-2.4keV) Beuermann 
\& Burwitz (1995) found that all systems showed large excesses. These authors claimed that for systems with magnetic field strengths $\gtrsim 30 \mathrm{MG}$ cyclotron emission dominates the emission from the shock: if this was taken into account then the excess would largely disappear. In contrast, Ramsay et al. (1994) did make an estimate of the observed cyclotron emission and include this in $L_{\text {shock }}$ but still found a significant number of systems with excesses.

To account for this excess, Kuijpers \& Pringle (1982) suggested that soft $\mathrm{X}$-rays could also be produced by dense blobs of material which penetrate into the photosphere of the white dwarf without forming a shock. There is also some evidence that the reprocessed component is emitted not as soft X-rays but in the extreme UV and therefore any distinct soft X-ray component is due to blobs of material (Heise \& Verbunt 1988). The energy balance in polars was therefore one of the prime areas for study using the XMM-Newton-MSSL survey.

\begin{tabular}{lr}
\hline Source & $L_{\text {soft }, \text { bol }} / L_{\text {hard }, \text { bol }}$ \\
\hline EU UMa (RE J1149+28) & 47 \\
V895 Cen & 13 \\
RX J1007-20 & 10 \\
RE J0501-03 & 3.8 \\
EK UMa & 1.7 \\
RX J1002-19 & 1.6 \\
EU Cnc & 1.2 \\
DP Leo & 1.1 \\
AN UMa & 1.0 \\
V347 Pav & 0.7 \\
EV UMa & 0.5 \\
BY Cam (soft-pole) & 0.5 \\
GG Leo (RX J1015+09) & 0.5 \\
RX J2115-58 (soft-pole) & 0.3 \\
EP Dra & 0.2 \\
RX J2115-58 (hard-pole) & - \\
WW Hor & - \\
CE Gru (Grus V1) & - \\
V349 Pav (V2009-65) & - \\
BY Cam (hard-pole) & - \\
V1500 Cyg & - \\
\hline
\end{tabular}

Table 1. The ratio $L_{s o f t, b o l} / L_{\text {hard,bol }}$ determined for those systems in a high accretion state.

In fitting the X-ray spectra we used a multi-temperature emission model to model the post-shock region (Cropper et al. 1999). This is in contrast to the ROSAT studies which used an (inappropriate) single temperature bremsstrahlung model. Some uncertainty arises as to the geometrical correction factor which should be applied to $L_{\text {soft }}$ - this is necessary since this component is optically thick. We have used a best estimate. We show in Table 1 the ratio $L_{\text {soft }} / L_{\text {hard }}$ for our current sample of polars which were found to be in a high accretion state. We find that very few systems show high ratios - only EU UMa, V895 Cen and RX J1007-20 do. Moreover, even without including the 
cyclotron component, we find that most systems are roughly consistent with the standard accretion model. A major surprise was that for 6 systems, at least one pole did not show evidence for a distinct soft X-ray component - the X-ray spectrum could be modelled with the multi-temperature emission model alone. These results are in contrast to that found in the ROSAT era. There are various reasons for the difference between the ROSAT and XMM-Newton results: the ROSAT energy band was not very sensitive to the hard X-ray component; in the ROSAT era a single temperature bremsstrahlung was used to model the hard component and the resolution of ROSAT was lower than XMM-Newton so it was more difficult to constrain the absorption model. To examine this in more detail, we have reanalysed the ROSAT data of one of the archtypical polars AN UMa (see Figure 2 for its XMM-Newton light curve). We compare the unabsorbed flux (rather than the luminosity, to make matters as simple as possible) of the soft and hard components using different models and satellite. For the multitemperature shock models we take the best fit parameters obtained using the $X M M-N e w t o n$ data and fix these (apart from the normalisation) when fitting the ROSAT spectra. The results are shown in Table 2.

We find that if we restrict the energy range to the ROSAT band (as in Beuermann \& Burwitz 1995), the resulting soft/hard ratio is typically much greater compared to using bolometric fluxes. Further, for the ROSAT data we find that using a one temperature shock model gives a greater ratio. However, perhaps the most interesting result is when we compare these ratios with those derived using XMM-Newton spectra. The soft/hard ratio determined over the XMMNewton energy range and their bolometric fluxes are much reduced compared to ROSAT data using every spectral model. Indeed, when we take geometrical factors into account to convert to luminosities, these ratios are consistent with the standard accretion model - even when we do not include a contribution from the cyclotron component. The effect of including a partial neutral absorption model is also noticeable. In the case of the XMM-Newton data the resulting mass of the white dwarf is lower when we include a partial covering component. The equivalent absorption column is also greater. Both these factors contribute to a higher soft/hard ratio.

Until now, one of the defining characteristics of polars has been the presence of a strong soft X-ray component. It is therefore surprising that we find 6 systems which showed at least one accretion pole which did not show a distinct soft X-ray component at all. It is likely that the re-processed component is cool enough to have moved out of the XMM-Newton band. In the scenario of Heise \& Verbunt (1988), those systems which show a soft component are accreting (at least some) dense blobs of material. However, for most of these systems the energy balance is consistent with the standard accretion model - which does not include blobs. Therefore for most systems where we detect a distinct soft X-ray component, but the ratio is compatible with that predicted by the standard model, the reprocessed component is in soft X-rays rather than the EUV as in the Heise \& Verbunt scenario. Clearly, we need a better understanding of the spectral characteristics of the re-processed component. The factors that may affect its temperature are $M_{w d}$ (since that sets the maximum temperature in the shock), $\dot{M}$ (since this sets the height of the shock) and $\mathbf{B}$ (since this sets amount of cooling due to cyclotron radiation). 


\begin{tabular}{|c|c|c|c|c|}
\hline Model & $\begin{array}{l}\text { flux } x_{\text {bolo,soft }} \\
\text { flux } \\
\text { bolo,hard } \\
\text { ROSAT }\end{array}$ & $\begin{array}{l}\text { flux } x_{\text {bolo,soft }} / \\
\text { flux bolo,hard } \\
\text { XMM-Newton }\end{array}$ & $\begin{array}{l}\text { flux }_{0.1-2.4, \text { soft }} \\
\text { flux } \\
\text { ROSAT }\end{array}$ & $\begin{array}{l}\text { flux }_{0.15-10, \text { soft }} \\
\text { flux } \\
\text { X.15-10,hard } \\
\text { XMM-Newton }\end{array}$ \\
\hline wabbbrem ${ }^{1}$ & 9.9 & 1.2 & 22.7 & 0.8 \\
\hline wabbbrem ${ }^{2}$ & 7.5 & 1.0 & 21.6 & 0.8 \\
\hline wabbsac & 2.3 & 1.4 & 7.7 & 0.9 \\
\hline wapcfbbsac & 5.2 & 3.0 & 7.6 & 1.4 \\
\hline
\end{tabular}

Table 2. The ratio of the unabsorbed flux in the soft and hard X-ray components derived for AN UMa in ROSAT and XMM-Newton using different spectral models. We show the bolometric flux and the flux over the ROSAT and XMM-Newton energy bands. wa refers to a neutral absorber, $p c f$ a neutral absorber with partial covering, $b b$ a blackbody, brem a thermal bremsstrahlung, and sac a multi-temperature accretion column. Note 1 refers to $30 \mathrm{keV}$, while 2 refers to $45 \mathrm{keV}$.

\section{Systems in a low accretion state}

One of the most surprising findings from our survey was that 15 out of the 33 currently observed polars in our sample were in low or much reduced accretion rates. Whether a system was in such a state was determined by comparing the $\mathrm{X}$-ray count rate with previous X-ray missions or from its long term optical light curve (since we have simultaneous UV-optical coverage).

To investigate this further we re-examined the fraction of all known polars that were in a high accretion state at the time of the ROSAT all-sky survey. Out of the 67 polars known at the end of 2002,25 were not discovered using the ROSAT all-sky survey. Of these systems 16 were found to be in a low accretion state. This is an even higher fraction than determined from our XMM-Newton survey. The fact that we observe around half or more polars in a low state is important when computing population models of polars.

Using our XMM-Newton sample, we tested whether those systems in a low accretion state were biased towards certain orbital periods. Using a rank test we find no evidence for high or low states correlating with period or preference. We also tested whether those systems which were in an off state (as opposed to those systems which were in low states but detectable) were biased: we find that there is marginal evidence for a bias towards shorter periods at the $91 \%$ confidence level.

Of the 15 systems detected in low or off states, 10 were detected in both the UVW1 and UVW2 filters in the optical/UV telescope. The UV fluxes remain approximately constant over the orbital cycle. It is therefore likely that we are detecting the white dwarf photosphere with little contribution from a heated accretion region. Work is in progress to determine their temperatures and compare them to temperatures derived in the high accretion state.

Although in low accretion states, a number of system showed evidence for a modulation in the X-ray light curves indicating that accretion was occuring at a low level. One system, ST LMi, showed evidence for accretion occuring during one orbital cycle, but not the previous cycle. The phasing is consistent with 

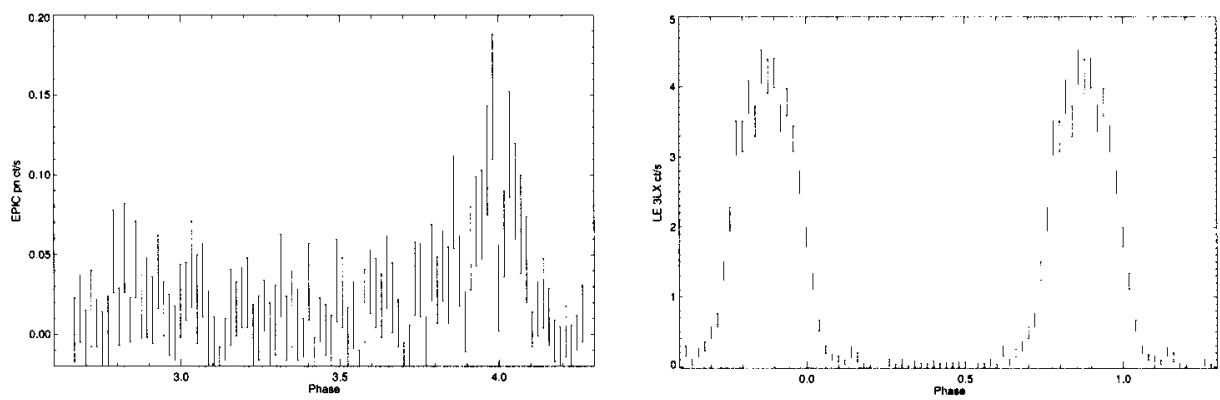

Figure 3. X-ray observations of ST LMi. Left panel: XMM-Newton observations, Right panel: EXOSAT observations.

accretion occuring onto the main accretion pole that has been seen to be X-ray bright at previous epochs (Figure 5). This spectrum could be modelled with a two-temperature thermal plasma model and the luminosity was $\sim 1 \times 10^{30}$ $\mathrm{erg} \mathrm{s}^{-1}$. This is similar with results of XMM-Newton observations of UZ For which also showed an accretion event (Still \& Mukai 2001, Pandel \& Còrdova 2002).

\section{References}

Beuermann, K. \& Burwitz, V., 1995, in Buckley, D. A. H., Warner, B., eds., Proc. Cape Workshop on Magnetic Cataclysmic Variables, ASP Conf. Series, 85,99

Cropper, M., Wu, K., Ramsay, G. \& Kocabiyik, A., 1999, MNRAS, 306, 684

Heise, J. \& Verbunt, F., 1988, A\&A, 189, 112

Jansen, F., Lumb, D., Altieri, B. (+12 co-authors), 2001, A\&A, 365, 1

Kuijpers, J. \& Pringle, J. E., 1982, A\&A, 114, L4

Lamb, D. Q. \& Masters, A. R., 1979, ApJ, 234. L117

Pandel, D., Còrdova, F. A., Shirey, R. E., Ramsay, G., Cropper, M., Mason, K. O., Much, R. \& Kilkenny, D., 2002, MNRAS, 332, 116

Pandel, D. \& Còrdova, F. A., 2002, MNRAS, 336, 1049

Ramsay, G., Mason, K. O., Cropper, M., Watson, M. G. \& Clayton, K. L., 1994, MNRAS, 270, 692

Ramsay, G., \& Cropper, M., 2002, MNRAS, 334, 805

Ramsay, G., \& Cropper, M., 2003, MNRAS, 338, 219

Schwope, A., Hambaryan, V., Schwarz, R., Kanbach, G. \& Gänsicke, B. T., 2002, A\&A, 392, 541

Still, M. \& Mukai, K., 2001, ApJ, 562, L71 